\title{
Cauchy Distribution
}

National Cancer Institute

\section{Source}

National Cancer Institute. Cauchy Distribution. NCI Thesaurus. Code C53219.

A continuous probability density distribution which models resonance behavior. It is also known as the Lorentz distribution. Its shape is dependent on a location parameter, which specifies the peak of the curve, and a scale parameter which specifies the half-width at half-maximum. 